\title{
Individual differences in event memory: A case for nonstrategic factors
}

\author{
RONALD L. COHEN \\ Glendon College, York University, Toronto, Ontario, Canada
}

\begin{abstract}
Individual differences in event free recall (episodic memory) and in item generation fluency (semantic memory) were investigated during the course of three studies. The findings suggested that, in addition to two specific factors, an episodic and a semantic factor, performance on both kinds of memory tasks had some dependency on a general factor. A case is made for interpreting all three factors as nonstrategic factors.
\end{abstract}

Kausler (1983) distinguished between rehearsaldependent and rehearsal-independent memory. These two classes of episodic memory may be classified according to the operations occurring during event storage. In rehearsal-dependent memory, events are stored through the aid of an encoding strategy, and the actual memorization operation (laying down of traces in long-term memory) is effortful. Rehearsal-independent memory refers to the traces of events that manage to find their way into long-term memory both effortlessly and without the aid of encoding strategies. The difference between the two classes of memory is confined to encoding, retrieval in both cases being characterized by effortful search. As Hasher and Zacks (1979) pointed out, the vast majority of laboratory research has been concerned with rehearsaldependent or strategic memory. In particular, the rather meager literature devoted to research into individual differences in episodic memory appears to have limited itself to strategic memory tasks. Consequently, it is hardly surprising that such differences have been attributed to effortful acquisition factors (Carroll, 1980).

Probably the most extensive investigation of individual differences in episodic memory (Underwood, Boruch, \& Malmi, 1978) used a factor-analytical approach set in the framework of an associationistic model. In that study, five episodic memory factors were isolated. Of particular interest here, free recall was found to load on one factor and paired associates and serial recall to load on a second factor. This result led Underwood et al. (1978) to speculate that, although the acquisition phases of free-recall and paired-associate tasks both involve the (presumably effortful) formation of associations, this process requires somewhat different abilities in the two cases. Items in a free-recall list may be acquired through the formation of contextual associations, whereas paired-associates learning presumably involves the formation of associations be-

This research was supported by Grant A7023 from the Natural Sciences and Engineering Research Council of Canada. Thanks are due J. M. Lacroix for his helpful comments on the manuscript. Reprint requests should be sent to Ronald L. Cohen, Department of Psychology, Glendon College, 2275 Bayview Avenue, Toronto, Canada, Ontario, M4N 3M6. tween the two items in each pair. In both cases, however, the conclusion is that individual differences in memory performance reflect individual differences in the rate at which associations are formed during acquisition. A follow-up study (Malmi, Underwood, \& Carroll, 1979) also found free recall and paired associates to load on two different factors, although serial recall changed its allegiance in this study and loaded on the free-recall factor. It should also be noted that the episodic memory measures in both studies tended to correlate with each other, suggesting the existence of a general episodic memory factor, namely, an association acquisition factor.

In addition to a large number of episodic tests, the Underwood et al. (1978) battery included three tests of semantic memory. Evidence for a dissociation between episodic and semantic memory was provided by the absence of correlations between the episodic and semantic memory scores. This latter result replicates an earlier finding (Anastasi, 1930) of a correlation between performance on two episodic tests, namely, paired associates and "retained members" (free recall), which did not extend to semantic memory performance. Clearly, semantic memory performance does not depend on the acquisition of episodic associations.

In a more recent study by Geiselman, Woodward, and Beatty (1982), individual differences in free recall were again attributed to effortful acquisition factors, namely, a major rehearsal factor and a minor intensity of processing factor. In particular, superior recall from long-term store was associated with semantic rehearsal strategies and high processing intensity.

An exception to this emphasis on effortful acquisition is provided by Robertson-Tchabo and Arenberg's (1976) study, which isolated a free-recall factor interpreted as a retrieval factor. Since there was nothing in the data of that study to support the choice of a retrieval over an acquisition explanation, however, little weight can be given to this interpretation.

The present investigation took the approach that, although effortful acquisition is undoubtedly important in some episodic memory tests, this concentration on effortful encoding may have obscured other equally important 
factors that contribute to individual differences. More specifically, we aimed to demonstrate the existence of a nonstrategic factor in episodic free recall. We also intended to investigate the possibility that if such a factor were isolated, it might well generalize to recall from semantic memory.

Before going on to describe our study, there are two features of previous studies that should be considered. First, earlier episodic memory studies have concentrated on the measurement of memory for verbal events, usually words. And second, performance on the particular semantic memory tasks used by Anastasi (1930) and by Underwood et al. (1978) could have been determined by the contents of the memory store (knowledge) or by proficiency in access (retrieval fluency). These two points will now be considered separately.

It is well established that the recall of verbal items is rehearsal dependent. In the case of words, for example, the recall probability of any given item depends on the number of rehearsals it has received (Rundus \& Atkinson, 1970), on the manner of its rehearsal (Craik \& Watkins, 1973; Geiselman et al., 1982), or, more generally, on the manner of its processing (Craik \& Tulving, 1975; Hyde \& Jenkins, 1969). Word recall is also affected by the use of mnemonics, such as chaining several unrelated words together to form a narrative (Bellezza, Richards, \& Geiselman, 1976), and by the use of composite imagery (Mueller \& Jablonski, 1970). Given that verbal events can be encoded in so many different ways and that recall level is strongly related to the manner of the encoding, it is hardly surprising that earlier individual difference studies have isolated strategic or effortful mnemonic factors.

The present study used a minibattery of tests to measure free-recall performance in adult samples. In order to minimize the role of effortful acquisition factors, this battery included an anchor test that is assumed to be nonstrategic in the sense that recall performance appears to have little connection with encoding strategies.

Partly to motivate the nonstrategic label, as applied to the anchor test, and partly to set the stage for the studies to be described here, a comparison will be drawn between some of the properties of the nonstrategic anchor test and those of word free recall, which is presumed to be strategic. The anchor test involves the free recall of series of actions performed by the subject (subject-performed tasks, or SPTs). These action events are presented by giving the subject a simple verbal instruction, such as "clap your hands" or "put the cap on the pen," and requiring him or her to enact the instructions. Subsequently, the subject free recalls the events.

A comparison of word and SPT properties yields the following picture. First, subjects report using active memorization strategies when presented with word lists, but not when presented with lists of SPTs. These subjective reports are consistent with the primacy effects obtained in word but not SPT recall (Cohen, 1981). Second, word recall is influenced by the levels-of-processing manipulation (Craik \& Tulving, 1975), whereas SPT recall apparently is not, this pointing to a flexibility in word encoding not shared by SPT encoding (Cohen, 1981). Third, designating words as being important to remember during acquisition greatly increases the likelihood of their being recalled relative to the likelihood of words not given an importance designation being recalled. This manipulation had only a minimal effect on SPT recall. Furthermore, if subjects are asked during acquisition to predict which of the presented items they will later recall, they are well able to do so in the case of words, but not in the case of SPTs. Again, later recall appears to be strongly related to control processes during acquisition in the case of words, but not in the case of SPTs (Cohen, 1983).

Individual differences data are also consistent with the strategic view of word recall and the nonstrategic view of SPT recall. Young children and retarded individuals are generally supposed to possess inferior encoding strategies (Belmont \& Butterfield, 1977; Brown, 1975; Campione \& Brown, 1978). Consequently, one property of a strategic memory test should be the presence of reliable age- and IQ-related differences. In line with our argument, reliable developmental differences have been found in the immediate free recall of words, but not of SPTs, in subjects ranging in age from 9 to 13 years (Cohen \& Stewart, 1982). The amount of variance in recall $\left(\right.$ omega $^{2}$ ) accounted for by the age variable was $44 \%$ in the word condition, but only $1 \%$ in the SPT condition. Similarly, a comparison between educable mentally retarded (EMR) and nonretarded subjects also showed a significant difference in the immediate free recall of words, but not of SPTs (Cohen \& Bean, 1983). In this case, the IQ variable accounted for $65 \%$ of the variance in the word recall, but for only $10 \%$ of the variance in the SPT recall. Furthermore, most of the IQ-related variance in the SPT condition had its source in the recall of recency items, which has in any case been shown to be far less dependent on acquisition strategies than is the recall of prerecency items (see, e.g., Glanzer, 1972, and Smith, Barresi, \& Gross, 1971).

At this stage in our researches, then, all the indications point to SPT recall's being more or less free from the influence of effortful encoding factors. Consequently, it is argued that any individual differences found in SPT recall performance reflect differences in storage beyond the control of the subject, or in retrieval.

The second of the aforementioned points involves the absence of a relationship between episodic and semantic recall performance found in prior studies. In the Underwood et al. (1978) study, semantic memory was tested with a vocabulary test (discriminating words from nonwords), a spelling test, and the verbal portion of the Scholastic Aptitude Test. In Anastasi's (1930) study, semantic memory was also tested with a vocabulary test, in this case forced-choice recognition of word meaning. Obviously, an incorrect response in any of these tests could depend on an access failure or on an insufficient knowledge base. In order to separate the access aspect 
of semantic memory from the knowledge aspect, our study used word-production tests. These tests are speed or fluency tests, rather than power tests: The subjects are required to produce as many words of a specified class as possible in a limited time interval (see Schonfield \& Stones, 1979). Although it cannot be claimed that performance on word fluency tests is completely independent of the contents of the lexical knowledge base, such tests are generally considered to be tests of retrieval efficiency (Burke \& Light, 1981; Schonfield \& Stones, 1979). It was hoped that the measurement of correlation coefficients between performance on episodic recall and performance on tests of semantic memory that emphasize retrieval fluency might uncover a general episodic-semantic recall factor not present in the earlier studies.

In accordance with our research objectives, we planned to test for the existence of an episodic memory factor common to a strategic test (free recall of words) and to the nonstrategic test constituted by the free recall of SPTs. Such a common factor would, of course, be indicated by the finding of a reliable positive correlation between word and SPT recall performance. It was planned that if such a relationship between the two episodic tests emerged in our first study, the possibility of this relationship's extending to semantic memory would be investigated in two further studies.

One prerequisite for the success of our researches was that the SPT test would yield reliable individual differences. Indeed, given the presumed nonstrategic nature of this task, there was a distinct possibility that the SPT test would not meet this prerequisite. Experiment 1 therefore tested for reliable individual differences in SPT recall and word recall, by measuring split-half reliabilities for freerecall performance on lists of SPTs and on lists of words. By testing the same subjects with both classes of events, it was also possible to calculate correlation coefficients between word and SPT recall performance.

Experiment 2 was essentially a replication of Experiment 1, with two modifications. The subjects in Experiment 1 were tested during the course of one session; in Experiment 2, the subjects were tested in two sessions, and reliabilities were measured as test-retest correlation coefficients. In addition, two tests of semantic memory were included in the test battery in Experiment 2.

Experiment 3 was a replication of Experiment 2, with two main modifications. First, the tests were administered to each subject during the course of one session. And second, the sample size was increased in order to allow a search for memory factors via a principal-components analysis.

\section{EXPERIMENT 1}

Experiment 1 was designed (1) to test for reliable individual differences in the free recall of word and SPT lists and (2) to test for reliable correlations between word and SPT recall.

\section{Method}

Materials. Eighty-four two-syllable words having a frequency of 20 or more per million (Kučera \& Francis, 1967) were selected from the Toronto word pool. These were assigned randomly to seven lists of 12 items.

Eighty-four tasks were selected from Cohen's (1981) task pool. These also were assigned randomly to seven lists of 12 items, with the constraint that tasks involving the use of objects alternated with tasks not requiring the use of objects. This constraint is generally used in SPT studies to facilitate the smooth presentation of these events.

These procedures were repeated a second time, giving two sets each of word lists and SPT lists. The word lists were audiotaped on a cassette recorder.

Subjects. Thirty-seven female volunteers from the Applied Psychology Unit subject panel in Cambridge, England, were tested in Experiment 1 . The ages in the sample lay in the range $25-49$ years. Twenty of these subjects were tested with one set each of SPT lists and word lists, in that order. The order of testing was reversed for the remaining 17 subjects, who first received the second set of word lists followed by the second set of SPT lists. Each subject was paid \$4 for her participation.

Procedure. For the SPT lists, the subject sat at a table, half of which was screened from her view. Some of the tasks involved the use of objects, whereas others did not. The objects required for task performance were hidden from the subject's view behind the screen. The experimenter presented each task by reading aloud a verbal instruction (e.g., "knock on the table"), following which the subject enacted the task. When the task required the use of an object, the object was presented together with the instruction; for example, the experimenter would present a toothpick with the instruction "Break the toothpick." Immediately following the task's completion, the experimenter retrieved the object, or parts thereof, and replaced it behind the screen. Following each list, the experimenter pointed to a pad in front of the subject to signal the subject to write down as many of the tasks as she could recall in any order.

In the word condition, the lists were simply played back to the subject. The end of each list was again signaled by the experimenter's pointing to the pad to initiate the subject's written free recall.

In both tests, the subjects were given modified free-recall instructions, in that they were informed that the optimal recall strategy involved recalling recency items first.

The rate of presentation was about 1 event $/ 5 \mathrm{sec}$ in the case of the SPT lists, which allowed sufficient time for the subject to perform the tasks with minimal delay between events. The rate of word presentation was matched with that of the SPTs, at 1 word $/ 5 \mathrm{sec}$. In the word condition, in which each event response was a single word, $60 \mathrm{sec}$ were allowed for recall. In the SPT condition, in which each event response consisted of a two- or three-word description, $80 \mathrm{sec}$ were allowed for recall.

\section{Results}

The first list in each condition was treated as a warmup trial. Table 1 gives the performance data for the remaining six lists, from which it is noted that the word condition yielded a greater spread of individual differences than did the SPT conditions.

Two performance scores were calculated for each subject under each condition, one score for Lists 2,4 , and 6 and one score for Lists 3, 5, and 7. The SpearmanBrown split-half reliabilities calculated from these scores are also shown in Table 1. As expected, word recall showed a high reliability in both replications. The coefficients were somewhat smaller in the SPT conditions, but sufficiently high to justify the conclusion that both word and SPT recall yielded reliable individual differences within the testing session. 
Table 1

Performance and Correlational Data (Experiment 1)

\begin{tabular}{|c|c|c|c|c|}
\hline & \multicolumn{2}{|c|}{$\begin{array}{c}\text { Replication } 1 \\
(\mathrm{~N}=17)\end{array}$} & \multicolumn{2}{|c|}{$\begin{array}{c}\text { Replication } 2 \\
(\mathrm{~N}=20)\end{array}$} \\
\hline & Words & SPTs & Words & SPTs \\
\hline Mean & 41.5 & 45.1 & 39.4 & 43.2 \\
\hline SD & 9.51 & 6.28 & 9.33 & 6.56 \\
\hline Split-Half & & & & \\
\hline Reliability & $.92^{*}$ & $.74^{*}$ & $.92 *$ & $.73 *$ \\
\hline Word/SPT & & & & \\
\hline Correlation & \multicolumn{2}{|c|}{$.50 *$} & \multicolumn{2}{|c|}{$.60^{*}$} \\
\hline
\end{tabular}

Correlations calculated between word and SPT performances based on recall from Lists 2 through 7 for each condition are given in the final row of Table 1 . In both replications, SPT and word recall show significant correlations.

As earlier indicated, Gieselman et al. (1982) reported that the free recall of words from long-term store was related to the type and intensity of rehearsal used during acquistion. This relationship did not, however, extend to recall from short-term store. The data reported in Table 1 are based on the subjects' total output from both shortterm and long-term stores. Consequently, the possibility must be considered that our correlations reflect mainly individual differences in recall from short-term store and therefore provide no information about the existence of a common factor in recall from long-term store. In order
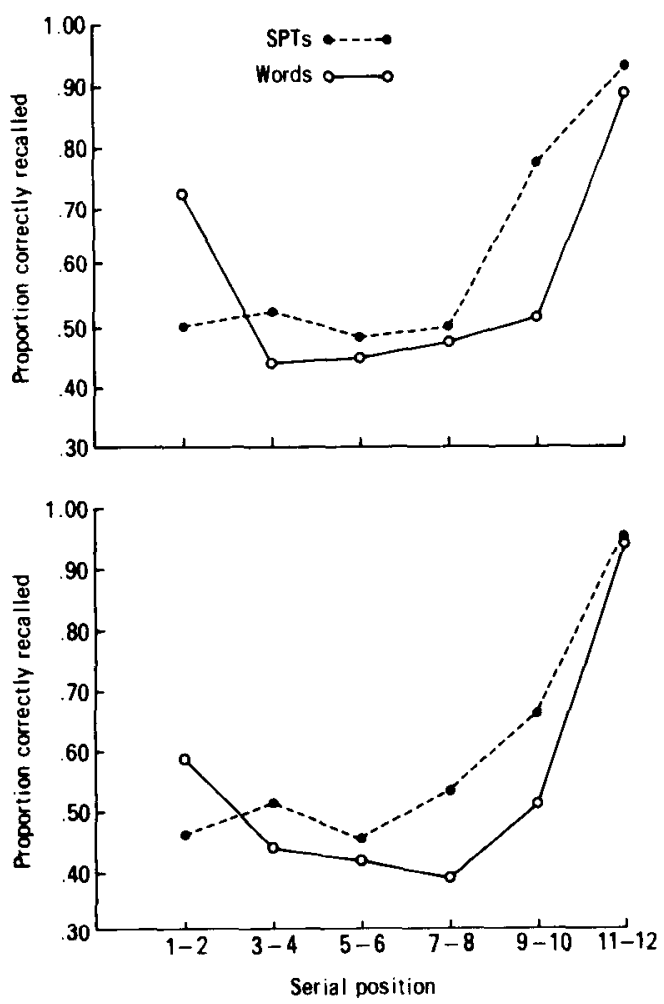

Figure 1. Serial position curves for the recall of words and SPTs in Experiment 1. As indicated, each data point represents recall from two adjacent serial positions. to examine this possibility, the data were rescored to give each subject two scores for each list, one for the recall of recency items and one for the recall of prerecency items. This division of performance into recency and prerecency recall should provide at least an approximate separation of recall from short-term and long-term stores.

The serial position curves for word and SPT recall are shown in Figure 1. In common with previous findings, word recall shows both recency and primacy; SPT recall shows only recency. For both types of items, it appears as though recency is confined to the final four events in the lists. The data were therefore rescored to give each subject one score for the first eight items and one for the final four items. Word-SPT correlations were then calculated for the recency and prerecency items separately. For the procedure in which the order of testing was SPTs followed by words, these correlation coefficients were .75 (prerecency) and .54 (recency); for the procedure in which the reverse order of testing was used, the coefficients were .48 (prerecency) and .30 (recency). Thus, decreasing the influence of the short-term recall component does not diminish the magnitude of the word-SPT correlations based on total list recall.

\section{Discussion}

In spite of our initial doubts, the SPT conditions yielded systematic individual differences. In fact, the amount of variance (reliability ${ }^{2}$ ) accounted for by the individual differences factor in SPT recall, approximately $55 \%$, was rather unexpected in light of the amounts of variance accounted for by the age variable in the Cohen and Stewart (1982) study (1\%) and by the IQ variable in the Cohen and Bean (1983) study (10\%). It would appear, then, that SPT recall depends to some extent on an ability that is distributed in the population, but that is minimally related to intellectual development, at least within the limits tested in our earlier studies. In line with our earlier argument, it is further concluded that this ability is not related to encoding strategies.

The correlations found between word and SPT recall suggest that although individual differences in word recall may reflect differences in the efficient use of encoding strategies, they also have some dependence on the nonstrategic factor assumed to underlie SPT recall.

\section{EXPERIMENT 2}

Since each subject in Experiment 1 was tested during the course of a single session, it is not possible to specify whether the common factor in SPT and word recall should be interpreted as reflecting a temporary state of the subjects or a more permanent ability. In order to resolve this question, the subjects in Experiment 2 free recalled word and SPT lists on each of two sessions, separated by an interval of at least 1 week. It was reasoned that if the common factor assumed to underlie the two tasks in Experiment 1 also manifested itself in the form of reliable testretest correlations in Experiment 2, then it could be con- 
cluded that the factor represented a relatively permanent ability.

In addition to the two episodic memory tests, Experiment 2 included two tests of guided word production in order to determine whether the memory factor shown to be common to the two episodic memory tests in Experiment 1 would extend its commonality to semantic memory tests.

\section{Method}

Materials. A pool of 96 words and a pool of 96 tasks, similar to those used in Experiment 1, were assembled. In each case the items were assigned randomly to eight lists of 12 events, again with the constraint in the SPT lists that tasks requiring the use of an object alternated with tasks that did not. Each of the two sets of eight lists was split randomly into two subsets of four lists, one for use on the first and the other for use on the second testing session.

The two semantic memory tests included in the test battery required the recall of single-word events. In one semantic test, recall was guided by a given initial letter, " $\mathrm{H}$ " on one session and " $\mathrm{F}$ " on the other. In the second test, recall was cued by category, "Cities" in one session and "Boys' Names" in the other. The test-retest constituents of the letter-prompted recall were equivalent in the sense that Webster's New World Dictionary (Guralnik, 1970) lists approximately equal numbers of words beginning with the letters " $\mathrm{H}$ " and " $\mathrm{F}$." For the categoryprompted recall, equivalence existed insofar as both test and retest required the recall of proper nouns.

Subjects. Eighteen female Glendon College undergraduates, in the age range 18-29 years, volunteered to serve as subjects. Each subject was paid $\$ 6$ for participating in the two sessions.

Procedure The word and task lists were presented and recalled as in Experiment 1, except for two minor modifications. Whereas the rate of SPT presentation was maintained at 1 event $/ 5 \mathrm{sec}$, the rate of word presentation was increased to 1 word $/ 2 \mathrm{sec}$. The second modification involved a change of instructions from modified free-recall instructions ("output final items first") to standard free-recall instructions ("recall in any order").

On the first session, each subject received the first subset of four word lists followed by the first subset of four SPT lists. Subsequent to these, two tests of semantic recall were administered. The first of these required the subject to recall as many words as possible beginning with the letter " $H$."' The second semantic test asked the subject to name as many cities as possible. In both cases, 2 min were allowed for written recall.

On the second session, the remaining subsets of four word lists and four SPT lists were presented, followed by the remaining two semantic tests, the first asking for words beginning with the letter " $F$ " and the second asking for boys' names.

\section{Results}

Table 2 gives the performance data and test reliabili-

Table 2

Performance Data and Test-Retest Reliabilities (Experiment 2)

\begin{tabular}{lccccc}
\hline & \multicolumn{2}{l}{ Episodic Memory } & \multicolumn{2}{c}{ Semantic Memory } \\
\cline { 3 - 5 } \cline { 5 - 5 } & Words & SPTs & $\begin{array}{c}\text { Letter-Cued } \\
\text { Words }\end{array}$ & $\begin{array}{c}\text { Category-Cued } \\
\text { Words }\end{array}$ \\
\hline Mean & 20.7 & 22.9 & 23.9 & 22.4 \\
SD & 4.50 & 3.24 & 6.47 & 6.28 \\
Mean & 19.3 & 24.5 & 26.2 & 31.5 \\
SD & 4.39 & 3.42 & 6.42 & 6.25 \\
Test-Retest & & & & \\
Reliability & $.80 *$ & $.52 *$ & $.82 *$ & $.69 *$ \\
\hline$* p<.05$. & & & &
\end{tabular}

Table 3

Intercorrelations Among the Four Memory Tests (Experiment 2)

\begin{tabular}{lccc}
\hline & SPTs & $\begin{array}{c}\text { Letter-Cued } \\
\text { Words }\end{array}$ & $\begin{array}{c}\text { Category-Cued } \\
\text { Words }\end{array}$ \\
\hline Words (free recall) & $.61^{*}$ & .37 & $.47^{*}$ \\
SPTs & & .36 & .34 \\
Letter-Cued Words & & & $.70^{*}$ \\
\hline
\end{tabular}

${ }^{*} p<.05$.

ties for Experiment 2. Test-retest reliabilities were calculated as correlation coefficients between the two word subtests, the two SPT subtests, the two letter-cued semantic memory tests, and the two category-cued semantic tests. As may be seen in Table 2, all of these tests showed reliable individual differences.

The word and SPT results were very similar to those obtained in Experiment 1, the SPT condition again yielding a narrower spread of scores and a lower reliability coefficient than the word condition.

In order to examine intercorrelations between the various pairs of test performances, the test and retest scores were summed to assign each subject one score for each of the four tests. These intercorrelations are given in Table 3. Although all the tests correlated positively with each other, only three of the correlations were large enough to reach the 05 significance level, namely, that between the two episodic tests, that between the two semantic tests, and that between episodic and category-cued word recall.

Finally, it should be noted that the serial position data replicated those found in Experiment 1, as did the relative strengths of the word/SPT correlations for recency recall $(\mathrm{r}=.22)$ and prerecency recall $(\mathrm{r}=.57)$.

\section{Discussion}

Although the two halves of the SPT and word tests were conducted at least 1 week apart in Experiment 2, the episodic memory data showed good replication of the data from Experiment 1, in which testing was conducted within a single session. It is therefore concluded that the factor isolated in Experiment 1 reflects a relatively permanent ability in the subjects, rather than a temporary state.

The data obtained from the semantic memory tests also suggest a relatively permanent ability common at least to these two tests. As in the case of the SPT/word episodic ability, it is difficult to argue that the semantic memory ability involves an acquisition strategy, and certainly not a test-specific acquisition strategy. For example, it is difficult, if not impossible, to imagine a situation in which the subjects had previously encoded " $H$ "- and " $F$ "-words into semantic memory according to their initial letters. This does not, of course, rule out the possibility that some subjects had acquired a larger vocabulary than others nor that some subjects had a greater knowledge of world geography or boys' names.

Even though several values in Table 3 are not significant, the general trend of positive correlations among the various tests suggests the possibility of a general memory factor. Given the relatively small sample size used in Experiment 2 , however, it was really difficult to draw any 
firm conclusion on this point. Partly for this reason, a modified replication of Experiment 2 was conducted with a larger subject sample.

\section{EXPERIMENT 3}

This experiment included the word and SPT recall tests and the letter-cued and category-cued semantic tests used in Experiment 2. One modification involved a reversion to the single session testing used in Experiment 1. A second modification involved the inclusion of a third semantic memory test. The two semantic memory tests in Experiment 2 resembled the episodic word recall test in that all three tests measured memory for word events. Experiment 3 included a semantic memory test that was regarded as an analogue to the episodic SPT test in that it required the recall of action events.

\section{Method}

Subjects. Forty female Glendon College undergraduates, in the age range $18-29$ years, volunteered to serve as subjects. Each was paid $\$ 5$ for her participation.

Materials. Seven word lists and seven SPT lists, each comprising 12 items, were constructed as in Experiment 1.

The letter-cued and category-cued semantic tests were similar to those used in Experiment 2. The third semantic test consisted of two subtests, one of which required the subject to list "things you do on vacation" and the other to list "things you do every day."

Procedure. Testing was similar to that in the previous two experiments. The order of testing for all subjects was word free recall, SPT free recall, recall of " $H$ "-words, recall of " $F$ "'-words, recall of cities, recall of boys' names, recall of vacation activities, and recall of everyday activities.

Rate of word presentation was 1 word $/ 2 \mathrm{sec}$; rate of SPT presentation was approximately $1 \mathrm{SPT} / 5 \mathrm{sec}$. Standard free-recall instructions were used. One minute was allowed for the recall of each word list, $80 \mathrm{sec}$ for the recall of each SPT list, and $2 \mathrm{~min}$ for recall in each of the semantic memory tests.

\section{Results}

Table 4 gives the performance data and test reliabilities for the five memory tests. Again, the similarity between these data and the data from the previous experiment (Table 2) is quite striking.

Again, the serial position data for the episodic tasks replicated those found in the earlier studies, word recall showing primacy and recency and SPT recall showing only recency. The word/SPT correlation was again higher for prerecency recall $(r=.49)$ than for recency recall $(\mathrm{r}=.31)$.

Table 5 shows the intercorrelations between the various pairs of tests. As in Experiment 2, all the tests show a tendency to correlate positively with one another. With

Table 4

Performance Data and Test Reliabilities (Experiment 3)

\begin{tabular}{lccccc}
\hline & \multicolumn{2}{c}{$\begin{array}{c}\text { Words } \\
\text { (Free Recall) }\end{array}$} & \multicolumn{4}{c}{ SPTs } & Wetter-Cued Category-Cued \\
& Words & Words & Activities \\
\hline Mean & 46.5 & 43.4 & 39.1 & 46.8 & 32.0 \\
SD & 9.18 & 4.14 & 10.02 & 10.57 & 7.54 \\
Reliability & $.91^{*}$ & $.50^{*}$ & $.93^{*}$ & $.77^{*}$ & $.76^{*}$ \\
\hline${ }^{*} p<.05$. & & & & &
\end{tabular}

the larger sample size, however, all except one of the episodic/semantic test correlations are now significant at the .05 level, which supports the notion of a general factor underlying all the memory tests in the battery. Indeed, a principal-components analysis performed on the correlation matrix yielded only one component having an eigenvalue $>1.0$ (the cutoff point for factor extraction, according to the Kaiser-Guttman rule). This component showed loadings in the range $.62-.82$ from all five tests, and accounted for $55 \%$ of the total variance.

Two further points of similarity are apparent between the Experiment 2 and the Experiment 3 data. The two episodic tests correlate more highly with each other than with the semantic tests; and the semantic tests correlate more highly with each other than with the episodic tests. These observations suggest that, over and above a general factor, there is the possibility of specific episodic and semantic factors. The principal-components analysis also supported this possibility by offering a second solution. If the Kaiser-Guttman rule is relaxed slightly, two factors may be extracted from the analysis, the second factor having an eigenvalue $=0.92$, and account for a further $18.2 \%$ of the common variance. (This violation of the KaiserGuttman rule is not entirely unwarranted, since the eigenvalue $>1.0$ criterion tends to be too strict when the analysis involves relatively few variables, as in the present case. See Cattell, 1966.) The result of this two-component extraction, followed by a varimax rotation, is given in Table 6 . The first component shows high loadings from all three semantic tests, but not from the episodic tests. The second component shows high loadings from the two episodic tests but not from the semantic tests.

Insofar as positive correlations are indicative of common underlying mechanisms, the results of Experiment 3 suggest a specific episodic factor and a specific semantic factor, in addition to a general memory factor. It should also be noted that Experiment 3 provides no support whatsoever for making a distinction between word event and action event memory. Indeed, the correlation between the two action event tests has the lowest value of all the coefficients in Table 5.

\section{Discussion}

It should be noted that although our episodic memory findings showed international replicability (England and Canada), the subjects in all three studies were exclusively female. Consequently, the following conclusions are, strictly speaking, valid only for female samples. There is, however, no obvious reason to expect male samples to produce different results.

Although the aim of our studies was to test for the possible existence of nonstrategic factors in memory, one prerequisite stated in the introduction was the finding of reliable individual differences in the presumed nonstrategic anchor test, namely, SPT recall. Our investigation clearly has demonstrated reliable individual differences in SPT recall, as well as in our other memory tasks. In addition, Experiment 2 has shown that these differences 
Table 5

Intercorrelations Among the Five Memory Tests (Experiment 3)

\begin{tabular}{lcccc}
\hline & SPTs & $\begin{array}{c}\text { Letter-Cued } \\
\text { Words }\end{array}$ & $\begin{array}{c}\text { Category-Cued } \\
\text { Words }\end{array}$ & Activities \\
\hline Words & & & & \\
(Free Recall) & $.48^{*}$ & $.40 *$ & $.39 *$ & $.39 *$ \\
SPTs & & $.32 *$ & $.38^{*}$ & .27 \\
$\begin{array}{l}\text { Letter-Cued } \\
\text { Words }\end{array}$ & & $.66^{*}$ & $.56^{*}$ \\
Category-Cued & & & $.52^{*}$ \\
Words & & & \\
${ }^{*} p<.05$. & & &
\end{tabular}

are not only reliable, but that they are also persistent, at least over a 7-day interval. This robustness of individual differences over time agrees with other reports of persistent individual differences in the memory literature. In particular, Woodhead and Baddeley (1981) reported persistent individual differences in facial recognition ability over intervals as long as 1 to 4 years.

Given that SPT performance meets our prerequisite, we can now proceed to review our data in relation to the main aims of our present studies. The two main questions posed in our present researches involved (1) the possibility of demonstrating a common nonstrategic factor underlying individual differences in episodic recall and (2) the possibility that any such factor would extend its influence to individual differences in semantic recall.

The results of Experiment 3 and, to a lesser extent, those of Experiment 2 support the notion of a general factor underlying both the episodic and semantic tests, in addition to the two specific factors. The interpretation of these factors, admittedly rather speculative, rests on two basic assumptions. The first assumption, which is surely unchallengeable, is that the acquisition of such events as activities people carry out every day or while on vacation are encoded nonstrategically. Consequently, any factor with a loading from the semantic "recall activities" tests should not be interpreted in rehearsal-dependent terms. The second assumption, which has a fair amount of empirical support, yields a similar case for any factor with a loading from the episodic SPT recall task.

In the case of the episodic factor, the choice appears to be between a nonstrategic acquisition factor and a retrieval factor. Opting for an acquisition interpretation of the episodic factor leads to a rather interesting and perhaps counterintuitive conclusion. Word recall, which is highly dependent on such factors as depth of encoding, encoding strategies, rehearsal, intensity of encoding, and encoding impact, shares a common factor with SPT recall, which exhibits none of these properties. In other words, interpreting the episodic, or, indeed, the general, factor as an acquisition factor strongly suggests that much of the individual differences in word recall (involving about $25 \%-35 \%$ of the variance) depends on rehearsalindependent acquisition. The alternative explanation for the word-recall/SPT-recall correlations, namely, individual differences in retrieval proficiency, is perhaps less counterintuitive, especially when considered in terms of Kausler's model (1983), which calls for similar retrieval operations in rehearsal-dependent and rehearsalindependent memory.

One obvious explanation for the semantic factor involves an exposure-acquisition explanation. High-scoring subjects are superior simply because they have been exposed to, and have therefore acquired, more words, boys' names, and cities than have low-scoring subjects. Although this is a possibility, it is difficult to understand why those same subjects have also been exposed to a greater number of everyday or vacation activities than the low scorers. Although the possibility of nonstrategic acquisition differences exists, the nature of the semantic tasks (speed tests) suggests that the semantic factor could be similar to the semantic access factor isolated by Hunt, Davidson, and Lansman (1981).

Finally, it remains to hypothesize about the nature of the general factor. Before doing so, it is perhaps instructive to compare our memory tests and findings with those of Underwood et al. (1978). A comparison of the semantic tasks used in our studies with those used in the Underwood et al. (1978) study clearly points to our tests' having the higher fluency component. Our tests limited the subjects' semantic recall to $2 \mathrm{~min}$; Underwood et al.'s subjects appear to have been given unlimited time to complete their semantic tasks. On the other hand, the Underwood et al. (1978) semantic tests obviously had a higher knowledge component than did our tests.

As mentioned above, it is difficult to imagine that our low performers actually had less knowledge of everyday or vacation activities than did our high performers. Consequently, our finding of a general episodic/semantic factor when semantic tasks that made optimal demands on speed but not on knowledge were used, contrasted with Underwood et al.'s failure to find such a factor when semantic tasks having knowledge but not speed demands were used, argues for our general factor's being a retrieval fluency factor, again akin to the semantic memory access factor isolated by Hunt et al. (1981).

Having argued for accepting at least the possibility that our factors may reflect individual differences in retrieval proficiency, we will now consider this possibility in further detail. Thus far, our use of the term nonstrategic has

Table 6

Component Loadings Following Varimax Rotation, From the Five Memory Tests in Experiment 3

Component 1

Component 2

Episodic

SPTs

Words

.31

.13

.78

Semantic

Words,

Letter-Cued

.81

.28

Words,

Category-Cued

.86

.19

Activities

.80

2.75

Eigenvalues

.16

.91 
referred to the actual memorization of the events. The obvious question, then, is whether the efficient use of strategies during retrieval is responsible for any of the common variance in our data. If this is the case, the types of retrieval strategies associated with success and failure on the various tests are not obvious from the subjects' protocols. Examination of the responses made by the subjects in the episodic recall tasks failed to reveal any obvious systematic strategies.

In the fluency tests, some subjects did show signs of strategy use. For example, in the word-production test, two subjects produced a cluster of words beginning with "ha" (or "fa") first, followed by a cluster of words beginning with "he," and so on. In the recall of cities, most subjects produced non-Canadian as well as Canadian cities and tended to cluster their output by country for North American cities and by continent for non-American cities. In the recall of everyday activities, six subjects tended to recall events in temporal order, from waking up in the morning to going to bed in the evening. Not only did the use of such organized retrieval appear to have no systematic effect on recall scores, but there was no discernible tendency for subjects to be general retrieval organizers in the sense that their protocols showed evidence of a retrieval strategy on more than one test.

Although the aforegoing observations are somewhat informal, they are at least suggestive that the use of a retrieval-strategy explanation for the factors isolated in our study would be inappropriate. This conclusion is supported in the case of the episodic factor by our earlier developmental and IQ studies, which failed to reveal reliable differences in SPT recall. Since both young children and retarded individuals should be penalized in any memory test favoring strategies, either encoding or retrieval, their demonstrated competence in SPT recall also argues against a retrieval-strategy explanation for any memory factor that includes a loading from the SPT test.

One further interpretation that should be considered stems from Underwood et al.'s (1978) suggestion that an important operation in episodic free recall involves the formation and use of item-context associations. Although the use of this notion to explain our specific episodic memory factor is quite consistent with our present data, there are other data that argue against it. Elderly individuals appear to possess inferior context-encoding skills (see, e.g., Kausler \& Puckett, 1981; see also the review in Burke \& Light, 1981). Elderly subjects do not, however, perform significantly worse than young adults in an SPT free-recall test (Bäckman \& Nilsson, 1984). Taken together, these two statements suggest that, whatever determines successful SPT recall, it is not related to context encoding.

In our introduction it was proposed that a bad memory might implicate factors beyond the subject's control. We submit that the results of our studies are consistent with this proposal. Not only can a strong case be made for considering the isolated factors, and especially the general factor, as being nonstrategic factors in the encoding sense, but it also appears that those factors are nonstrategic in the retrieval sense.

As already mentioned in the introduction, earlier descriptions of individual differences in episodic word recall have stressed effortful acquisition or encoding operations (Carroll, 1980; Geiselman et al., 1982; Underwood et al., 1978). Of course, none of these descriptions is incompatible with our data. The reliable variance associated with the individual differences in word recall accounted for in our studies (reliability ${ }^{2}$ ) range from $64 \%$ to $85 \%$. Since the variances common to word and SPT recall lie in the 23\%-37\% range, this leaves a fair amount of reliable variance to be accounted for in the word-recall data, presumably by strategic encoding factors. In this connection, it is of interest to note that in the case of the SPT recall, which is presumed to be nonstrategic, there is considerably less reliable surplus variance specific to the SPT test. In fact, in Experiment 3, SPT recall correlates with word recall about as highly as it correlates with itself.

Finally, the results of Experiments 2 and 3 can be considered in the light of the present controversy surrounding the episodic/semantic memory distinction. Although there is some disagreement as to whether semantic and episodic memory are functionally distinct (see, e.g., Anderson \& Ross, 1980, McCloskey \& Santee, 1981, and Muter, 1978), Tulving (1983) claimed that there is a general agreement for making a heuristic distinction between the two types of memory. As part of this general agreement, Tulving (1983) cited the absence of a semantic-episodic relationship as reported in Underwood et al.'s (1978) study. Although our present finding of a general fluency factor spanning all our memory tests may not constitute strong evidence against making a distinction, heuristic or otherwise, between semantic and espisodic memory, it does provide a counterargument to the use of the Underwood et al. finding in the support of the distinction. That the results of Experiment 3 and, to a lesser extent, those of Experiment 2 also suggest specific semantic and episodic memory factors is hardly surprising. Given the differences in properties shown by these two classes of memory test (Tulving, 1983), a failure to find some measure of factorial separation between semantic and episodic performance would have indeed been surprising.

\section{REFERENCES}

ANASTASI, A. (1930). A group factor in immediate memory. Archives of Psychology, 18, 1-61.

ANDERSON, J. R., \& Ross, B. H. (1980). Evidence against a semanticepisodic distinction. Journal of Experimental Psychology: Human Learning and Memory, 6, 441-465.

BäcKman, L., \& NiLsson, L.-G. (1984). Aging effects in free recall: An exception to the rule. Human Learning, 3, 53-69.

Bellezza, F. S., Richards, D. L., \& Geiselman, R. E. (1976). Semantic processing and organization in free recall. Memory \& Cognition, $4,415-421$ 
Belmont, J. M., \& Butterfield, E. C. (1977). The instructional approach to developmental cognitive research. In R. V. Kail \& J. W. Hagen (Eds.), Perspectives on the development of memory and cognition. Hillsdale, NJ: Erlbaum.

Brown, A. L. (1975). The development of knowing: Knowing, knowing about knowing, and knowing how to know. In $\mathrm{H}$. W. Reese (Ed.), Advances in child development and behavior (Vol. 10). New York: Academic Press.

Burke, E. M., \& Light, L. L. (1981). Memory and aging: The role of retrieval processes. Psychological Bulletin, 90, 513-546.

CAmpione, J. C., \& Brown, A. L. (1978). Toward a theory of intelligence: Contributions from research with retarded children. Intelligence, 2, 279-304.

CARROLL, J. B. (1980). Individual difference relations in psychometric and experimental cognitive tasks (Rep. No. 163). University of North Carolina, L. L. Thurstone Psychometric Laboratory.

CATtell, R. B. (1966). The scree test for the number of factors. Multivariate Behavioral Research, 1, 245-276.

CoHen, R. L. (1981). On the generality of some memory laws. Scandinavian Journal of Psychology, 22, 267-281.

CoHEN, R. L. (1983). The effect of encoding variables on the free recall of words and action events. Memory \& Cognition, 11, 575-582.

Cohen, R. L., \& BeAN, G. (1983). Memory in educable mentally retarded adults: Deficit in subject or experimenter? Intelligence, 7 , 287-298.

Cohen, R. L., \& Stewart, M. (1982). How to avoid developmental effects in free recall. Scandinavian Journal of Psychology, 23, 9-16.

Craik, F. I. M., \& Tulving, E. (1975). Depth of processing and the retention of words in episodic memory. Journal of Experimental Psychology: General, 104, 268-294.

Craik, F. I. M., \& Watkins, M. J. (1973). The role of rehearsal in short-term memory. Journal of Verbal Learning and Verbal Behavior, 12, 599-607.

Geiselman, J., WoOdward, A., \& BeatTy, J. (1982). Individual differences in verbal memory performance: A test of alternative informationprocessing models. Journal of Experimental Psychology: General, 111, 109-134.

GLANZER, M. (1972). Storage mechanisms in recall. In G. H. Bower (Ed.), The psychology of learning and motivation: Advances in research and theory (Vol. 5). New York: Academic Press.

GuRALNIK, D. B. (Ed.-in-chief). (1970). Webster's New World Dictionary (2nd. ed.). Toronto: Nelson, Foster \& Scott.

HASHER, L., Zacks, R. T. (1979). Automatic and effortful processes in memory. Journal of Experimental Psychology: General, 108 , 356-388.
Hunt, E. B., Davidson, J., \& Lansman, M. (1981). Individual differences in long-term memory access. Memory \& Cognition, 9, 599-608.

Hyde, T. S., \& Jenkins, J. J. (1969). Differential effects of incidental tasks on the organization of recall of a list of highly associated words. Journal of Experimental Psychology, 82, 472-481.

Kausler, D. H. (1983, August). Episodic memory and human aging. Paper presented at the American Psychological Association meeting, Anaheim, CA.

Kausler, D. H., \& Puckett, J. M. (1981). Adult age differences in memory for sex of voice. Journal of Gerontology, 36, 44-50.

KučEra, H., \& Francis, W. N. (1967). Computational analysis of present-day American English. Providence: Brown University Press.

Malmi, R. A., UNDERwood, B. J., \& CARRoll, J. B. (1979). The interrelationships among some associative learning tasks. Bulletin of the Psychonomic Society, 13, 121-123.

MCCloskey, M., \& SANTEe, J. (1981). Are semantic memory and episodic memory distinct systems? Journal of Experimental Psychology: Human Learning and Memory, 7, 66-71.

MUELLER, J. H., \& JABLONSKI, E. (1970). Instructions, noun imagery and priority in free-recall. Psychological Reports, 27, 559-566.

MuTER, P. (1978). Recognition failure of recallable words in semantic memory. Memory \& Cognition, 6, 9-12.

Robertson-TChabo, E. A., \& ARENBERG, D. (1976). Age differences in cognition in healthy educated men: A factor analysis of experimental measures. Experimental Aging Research, 2, 75-79.

Rundus, D., \& ATKINSON, R. C. (1970). Rehearsal processes in freerecall: A procedure for direct observation. Journal of Verbal Learning and Verbal Behavior, 9, 99-105.

SCHONFIELD, D., \& STONES, M J. (1979). Remembering and aging. In J. F. Kihlstrom \& F.J. Evans (Eds.), Functional disorders of memory. Hillsdale, NJ: Erlbaum.

Smith, E. E., Barresi, J., \& Gross, A. E. (1971). Imaginal versus verbal coding and the primary-secondary memory distinction. Journal of Verbal Learning and Verbal Behavior, 10, 597-603.

Tulving, E. (1983). Elements of episodic memory. Oxford: Clarendon Press.

Underwood, B. J., Boruch, R. F., \& Malmi, R. A. (1978). The composition of episodic memory. Journal of Experimental Psychology: General, 107, 393-419.

WOODHEAD, M. M., \& BADDELEY, A. D. (1981). Individual differences and memory for faces, pictures and words. Memory \& Cognition, 9 , $368-370$

(Manuscript received May 21, 1984; revision accepted for publication June 12, 1984.) 